\title{
If a researcher disagrees with a veterinarian's recommendations, whose say goes?
}

\author{
P \\ rofessor Archibald Boyd was a noted \\ primate neurophysiologist and clinical \\ neurologist at Great Eastern University. \\ His research focused on single-cell brain \\ recordings from macaques that had been \\ surgically fitted with metal implants attached \\ to their skull. As part of the school's every \\ third-year protocol renewal requirement, \\ Boyd's IACUC protocol was reviewed \\ by one of the school's veterinarians. The \\ veterinarian, with the agreement of the other \\ veterinarians, recommended a three-drug \\ multimodal surgical analgesia regimen \\ consisting of buprenorphine (an opioid), \\ a local nerve block with bupivacaine, and \\ meloxicam, a nonsteroidal anti-inflammatory \\ drug (NSAID). Boyd's original protocol \\ used only buprenorphine for postoperative \\ pain relief. The veterinarian noted that the \\ procedures Boyd used required significant \\ muscle dissection and periosteal disruption, \\ and even placement of a monkey's head in the \\ needed stereotactic apparatus could be painful.
}

The veterinarian added that the multimodal analgesia being recommended would bring Boyd's protocol in line with similar protocols now used with monkeys at Great Eastern and would be consistent with standard practice used at several primate centers and universities.

Boyd resubmitted his protocol without the recommended additional analgesics and without indicating if there was any scientific rationale for avoiding the NSAIDs or nerve block, such as interference with his data. However, he did write that he believed the additional drugs were unnecessary because his animals always looked good the morning after surgery, consistent with what would be expected after a long procedure. And, he added, the procedure itself was little more than a skin incision, some muscle dissection, and fixing the apparatus to the skull. He claimed that the Guide for the Care and Use of Laboratory Animals ${ }^{1}$ and the Animal Welfare Act's regulations ${ }^{2}$ did not require multimodal analgesia, and that the veterinarian's role was limited to consultation and advisement. Although the IACUC had approved multimodal analgesic treatment in other primate protocols, it agreed that it should not force an investigator to follow a veterinarian's recommendations. After a full-committee discussion, the protocol was approved as resubmitted by Boyd.

What is your opinion of the IACUC's actions?

\section{Jerald Silverman \\ University of Massachusetts Medical School, \\ Worcester, MA, USA. \\ e-mail: Jerald.Silverman@umassmed.edu}

Published online: 18 December 2019 https://doi.org/10.1038/s41684-019-0443-2

References

1. Institute for Laboratory Animal Research. Guide for the Care and Use of Laboratory Animals, 8th ed. (National Academies Press, Washington D.C., 2011).

2. Animal Welfare Act Regulations. 7 U.S.C. 2131-2159; 7 CFR 2.22, 2.80 , and 371.7

\section{IACUC fails to remember veterinarian as SME}

T he IACUC cannot simply state they "agree that it should not force an investigator to follow a veterinarian's recommendations," as it is the job of the attending veterinarian $(\mathrm{AV})$ to ensure the appropriate use of analgesia ${ }^{1,2}$. The AV is the Subject Matter Expert (SME) on appropriate analgesics in postsurgical animals. Although the veterinarian did not make a strong argument for changing the analgesic protocol, as stating "other protocols are doing this" is not a justification for changing this particular one, it is a reason to discuss the potential changes; Boyd should have acknowledged this. The veterinarians' proposal would have been stronger had they referenced historical assessment of Boyd's post-op animals as well as current methods-their approach is a refinement of analgesia and one that the IACUC should have considered more seriously.

Boyd's assessment of his post-op animals is based on observations collected on the following morning and says nothing about the immediate post-operative period-the period of time between recovery from anesthesia and the next morning, which could be anywhere from 12-18 hours after the administration of analgesic - and his statement about previous pain control being sufficient has not been adequately proven. The veterinarians' suggestions for a local nerve block, which provides immediate pain control up to several hours post-op, and NSAID, which provides for reduction in inflammation as well as swelling associated with surgical trauma, would be a welcome adjunct to an opioid analgesic. In fact, our personal experience has been that NSAIDs reduce the need to use a controlled substance such as buprenorphine. In other words, the proposed changes in analgesia, as suggested by the AV, would benefit Boyd's post-operative care and potentially improve the well-being of his research animals. Had he done his due diligence in searching the literature, he might have discovered this. Boyd failed to justify the refusal upon scientific grounds, which should have been reason for the IACUC to deny his proposal.

The IACUC may require that Boyd do further research into appropriate pain control as this area has been evolving. What worked in the past isn't always appropriate. Without scientific data that supports his resistance to change, Boyd cannot justify his analgesic regimen, particularly as other institutions develop further refinements that need to be assessed with respect to his own research.

In an ideal situation, both Boyd and the IACUC could have discussed the researcher's needs and historical assessment of the nonhuman primates, with a greater understanding of the timing around postoperative assessments and how they relate to the analgesics used. They could have also accepted the changes in current analgesic treatments, and even added the recommended analgesics as alternatives in situations where animals were noted to be potentially in pain or distress, so those analgesics could be used without having to wait for approval.

\section{Ginger Tansey}

National Eye Institute, National Institutes of Health, DHHS, Bethesda, MD, USA.

e-mail:tanseyg@nei.nih.gov

Published online: 18 December 2019 https://doi.org/10.1038/s41684-019-0440-5

\footnotetext{
References

1. 9 CFR Part $2 \$ 2.31$ Institutional Animal Care and Use Committee (d) (iv) (A) and (B)
}

2. 9 CFR Part $2 \$ 2.33$ Attending Veterinarian (b) (4) 